\title{
Successfull challenging percutaneous coronary interventions in two patients with anomalous left coronary artery origin from the right sinus of Valsalva
}

\author{
Sabri Demircan, Serkan Yüksel* \\ Department of Cardiology, Faculty of Medicine, Ondokuz Mayıs University, Samsun, Turkey
}

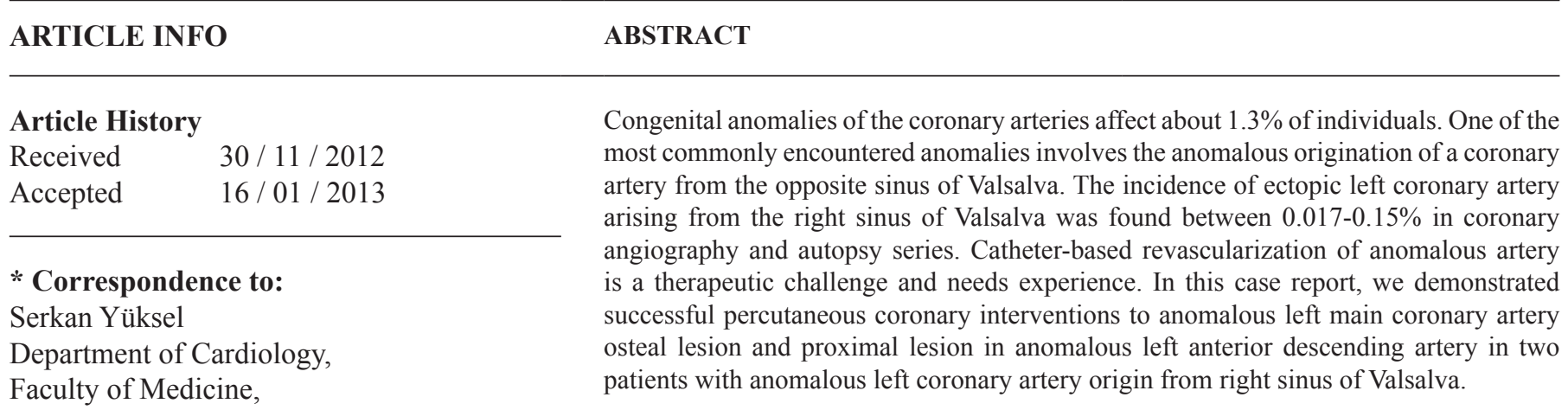

Ondokuz Mayıs University,

Samsun, Turkey

J. Exp. Clin. Med., 2013; 30:93-95

e-posta: serkan.yuksel@omu.edu.tr

\section{Keywords:}

Anomalous left coronary artery

Coronary artery disease

Congenital coronary anomaly

Percutaneous coronary intervention

\section{Introduction}

Congenital anomalies of the coronary arteries affect about 1.3\% of individuals (Engel et al., 1975; Chaitman et al., 1976; Baltaxe and Wixson, 1977; Kimbiris et al., 1978; Levin et al., 1978; Liberthson et al., 1979; Yamanaka and Hobbs, 1990; Garg et al., 2000). One of the most commonly encountered potentially serious anomalies involves the anomalous origination of a coronary artery from the opposite sinus of Valsalva. The prevalence of coronary disease in patients with the anomalous coronary arteries was not significantly different from patients without anomalies, with the exception of a significantly greater stenosis in anomalous circumflex $(\mathrm{Cx})$ arteries than in control arteries (Click et al., 1989). Catheterbased revascularization of anomalous coronary arteries is a therapeutic challenge for interventional cardiologists. In this case report, we demonstrated successful percutaneous coronary intervention to left main coronary artery (LMCA) osteal lesion and lesion in the left anterior descending (LAD) artery in two patients with anomalous left coronary artery origin from right sinus of Valsalva (RSV).

\section{Case 1}

An eighty two years-old female patient admitted to emergency department with the complaint of chest pain that started 3 days ago. Chest pain was squeezing and radiating to the back and lasting for 5 to 15 minutes. Physical examination and vital signs were normal. Initial electrocardiography (ECG) was in sinus rhythm with left bundle brunch block. Cardiac markers were elevated (CK:389 U/L, CK-MB: $58 \mathrm{ng} / \mathrm{mL}$ and Troponin-I: $9.8 \mathrm{ng} / \mathrm{L}$ ). After the initial evaluation she had the diagnosis of non-ST elevation myocardial infarction and transferred to coronary intensive care unit. On transthoracic echocardiography, left ventricle dimensions were normal but there was hypokinesia on apex, anterior and lateral walls of left ventricle and also her left ventricular ejection fraction was $30 \%$ by Simpson method. The next day, she was taken to catheterization laboratory. Coronary angiography showed a significant (90\%) stenosis on LMCA which had an abnormal origin from RSV (Fig. 1). The right coronary artery (RCA) also originated from the same sinus and had no stenosis. Percutaneous coronary intervention to LMCA was planned at the 


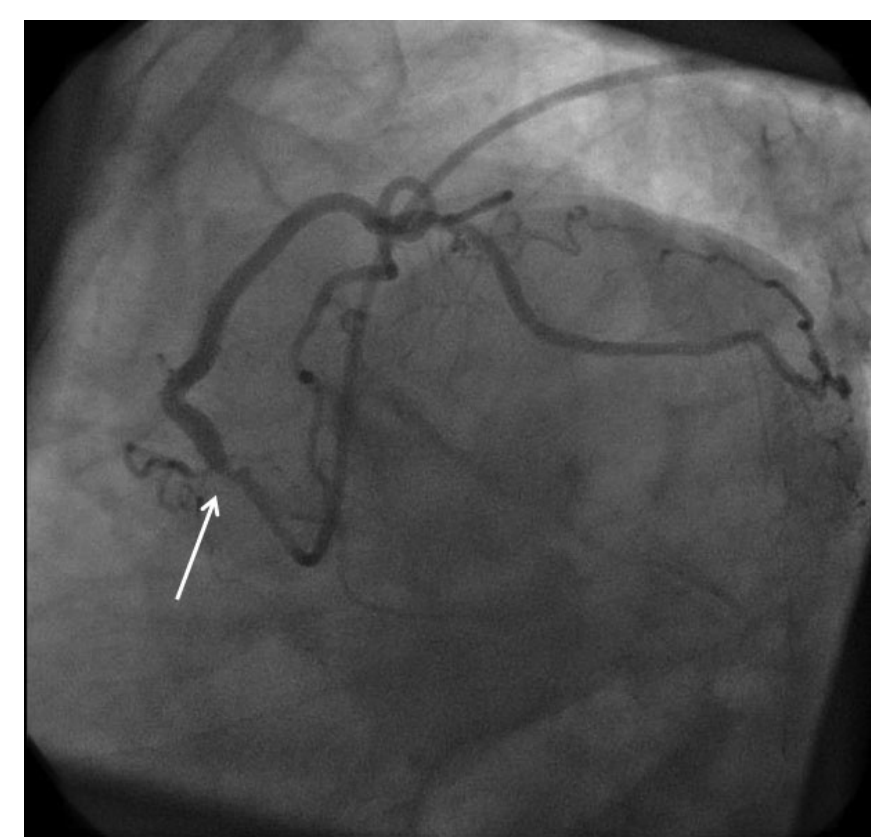

Fig. 1. Anomalous origin of left coronary artery (LCA) from right sinus of Valsalva and severe stenosis of left main coronary artery (LMCA) (shown by arrow).

same session. After a predilatation with $2.5 \times 10 \mathrm{~mm}$ balloon, a $3.5 \times 12 \mathrm{~mm}$ drug eluting stent was successfully implanted (Figure 2). After 48 hours of uncomplicated inpatient follow up, patient was discharged.

\section{Case 2}

A seventy seven years-old female patient admitted to cardiology outpatient clinic with the complaint of chest pain. It was atypical. But it had increased in intensity and duration since last week. She was diabetic and hypertensive for 10 years. Her physical examination and ECG was normal. An exercise stress test was performed and it was positive. Coronary angiography was planned and performed. Coronary angiography revealed a congenital coronary anomaly that all the coronary arteries were originated from RSV (Fig. 3). There were significant stenosis at proximal LAD and posterolateral branch of the RCA which is a dominant artery. A 4.0x13.0 $\mathrm{mm}$ bare metal stent to LAD and $2.75 \times 15 \mathrm{~mm}$ drug eluting stent to posterolateral branch of RCA was implanted (Figure 4). Procedure was completed successfully and patient was discharged without any complication.

\section{Discussion}

Congenital anomalies of the coronary arteries affect about 1.3\% (range, 0.2-5.6\%) of individuals (Engel et al., 1975; Chaitman et al., 1976; Baltaxe and Wixson, 1977; Kimbiris et al., 1978; Levin et al., 1978; Liberthson et al., 1979; Yamanaka and Hobbs, 1990; Garg et al., 2000). Approximately $80 \%$ of anomalies are considered benign without significant clinical sequel; the remaining $20 \%$ can potentially cause symptoms and may be responsible for significant disease (Yamanaka and Hobbs, 1990). One of the most commonly encountered potentially serious anomalies involves the anomalous origination of a coronary artery from the opposite sinus of Valsalva. Either the RCA or the left coronary artery (LCA) can arise from the opposite sinus and then traverses across the heart to resume a normal position. The incidence of ectopic LCA arising from the RSV was found between $0.017-0.15 \%$

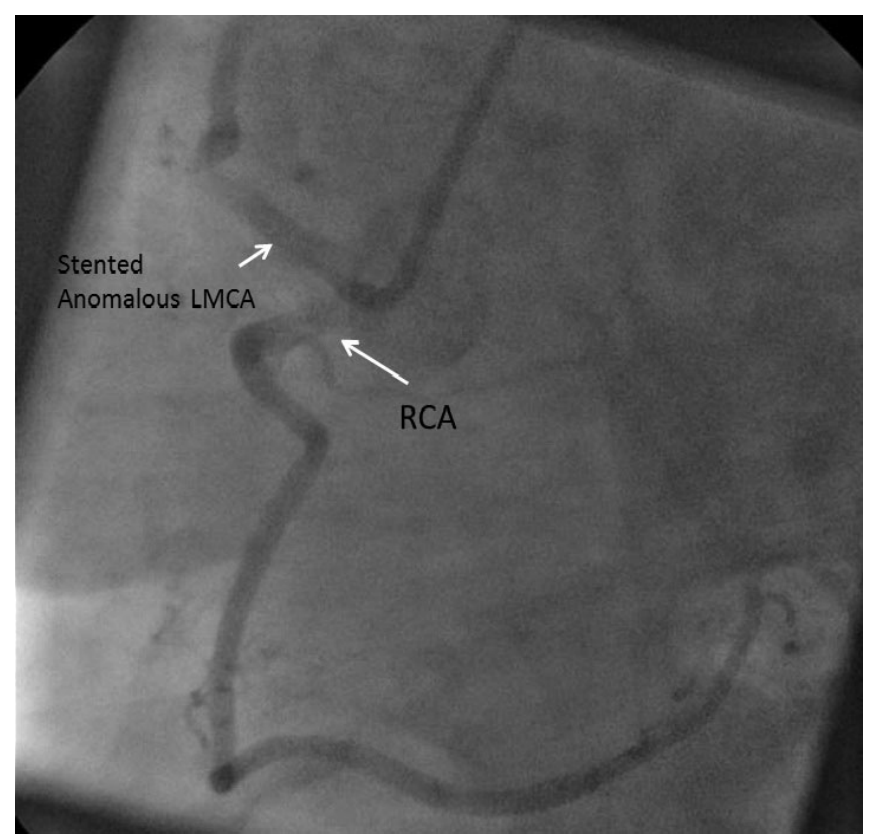

Fig. 2. Anomalous origin of left coronary artery (LCA) and right coronary artery (RCA) from the right sinus of Valsalva (RSV) and stented left main coronary artery (LMCA).

in coronary angiography and autopsy series (Yamanaka and Hobbs, 1990; Garg et al., 2000). There are several potential courses for an anomalous LCA arising from the RSV or the RCA. Diagnosis of the exact course is important because it will determine if intervention is necessary. There are four possible pathways for the anomalous LCA; between the aortic root and the pulmonary artery (interarterial course), a trans-septal (intraseptal or subpulmonic) course, anterior to the right ventricular outflow tract (anterior or prepulmonic course), and posterior to the aortic root (retroaortic course). Although the anterior, posterior, and septal (subpulmonic) courses are benign, an interarterial course carries a high risk for sudden cardiac death (Cheitlin et al., 1974; Earls, 2006).

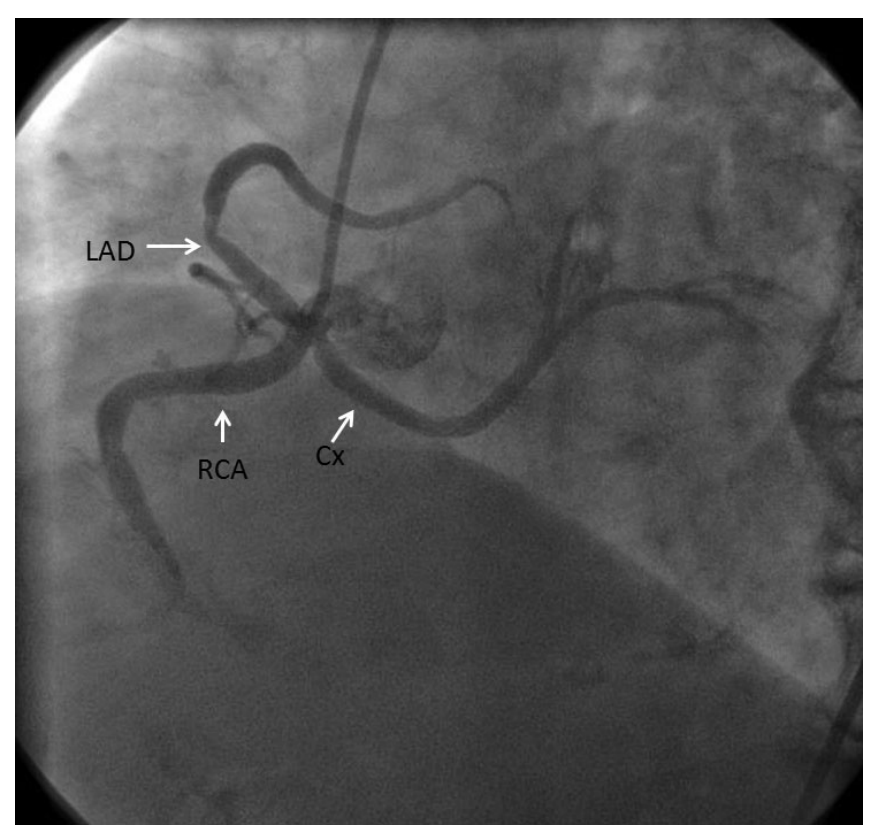

Fig. 3. Anomalous origin of left anterior descending (LAD), circumflex $(\mathrm{Cx})$ and right coronary arteries (RCA) from right sinus of Valsalva (RSV). 


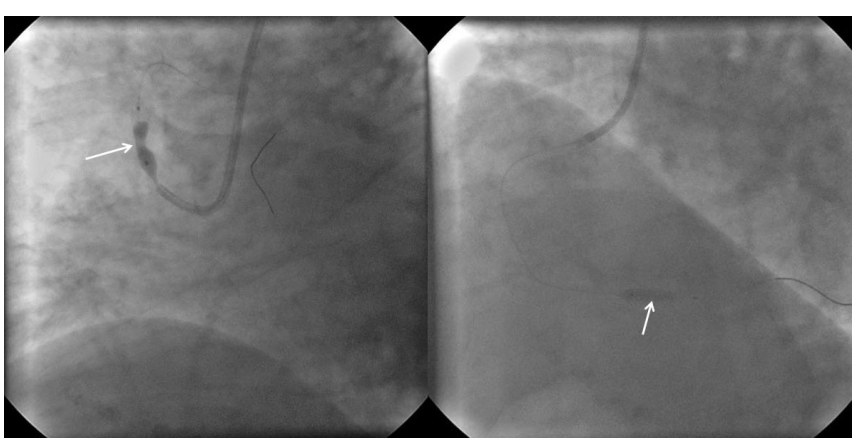

Fig. 4. Stent implantation to lesions in the proximal left anterior descending (LAD) artery and posterolateral branch of right coronary artery (RCA).

Click et al. (1989) reported that the degree of stenosis in the anomalous coronary arteries was not significantly different than that in the arteries of matched control patients without anomalies, with the exception of a significantly greater stenosis in anomalous $\mathrm{Cx}$ arteries than in control arteries. Catheter-based revascularization of this anomaly is a therapeutic challenge for interventional cardiologists. Percutaneous coronary intervention of an anomalous coronary artery requires proper angiographic recognition of the anatomic details, such as the orifice configuration, exit angulation, and the route of the anomalous artery. In selected patients, a lesion in a LMCA of anomalous origin can be treated safely and successfully with percutaneous coronary intervention (Mirchandani and Phoon, 2005; Nef et al., 2007; Tejada et al.,2007).

In conclusion, catheter-based revascularization of an anomalous coronary artery is a therapeutic challenge and needs experience. An interventional cardiologist must be aware of the complications and risks of performing such procedures in this group of patients, but coronary angioplasty with stenting may be a feasible therapeutic option, especially when an early revascularization may improve the patient outcome.

\section{REFERENCES}

Baltaxe, H.A., Wixson, D., 1977. The incidence of congenital anomalies of the coronary arteries in the adult population. Radiology 122, 47-52.

Chaitman, B.R., Lesperance, J., Saltiel, J., Bourassa, M.G., 1976. Clinical, angiographic, and hemodynamic findings in patients with anomalous origin of the coronary arteries. Circulation. 53, 122-131.

Cheitlin, M.D., De Castro, C.M., McAllister, H.A., 1974. Sudden death as a complication of anomalous left coronary origin from the anterior sinus of Valsalva: A not-so-minor congenital anomaly. Circulation. 50, 780-787.

Click, R.L., Holmes, D.R., Vlietstra, R.E., Kosinski, A.S., Kronmal, R.A., 1989. Anomalous coronary arteries: Location, degree of atherosclerosis and effect on survival-a report from the Coronary Artery Surgery Study. J. Am. Coll. Cardiol. 13, 531-537.

Earls, J.P., 2006. Coronary artery anomalies. Tech Vasc Interv Radiol. 9, 210-217.

Engel, H.J., Torres, C., Page, H.L., 1975. Major variations in anatomical origin of the coronary arteries: Angiographic observations in 4,250 patients without associated congenital heart disease. Catheter Cardiovasc. Diagn. 1, 157-169.

Garg, N., Tewari, S., Kapoor, A., Gupta, D.K., Sinha, N., 2000. Primary congenital anomalies of the coronary arteries: A coronary arteriographic study. Int. J. Cardiol. 74, 39-46.

Kimbiris, D., Iskandrian, A.S., Segal, B.L., Bemis, C.E., 1978. Anomalous aortic origin of coronary arteries. Circulation. 58, 606-615.

Levin, D.C., Fellows, K.E., Abrams, H.L., 1978. Hemodynamically significant primary anomalies of the coronary arteries. Angiographic aspects. Circulation. 58, 25-34.

Liberthson, R.R., Dinsmore, R.E., Fallon, J.T., 1979. Aberrant coronary artery origin from the aorta. Report of 18 patients, review of literature and delineation of natural history and management. Circulation. 59, 748-754.

Mirchandani, S., Phoon, C.K., 2005. Management of anomalous coronary arteries from the contralateral sinus. Int. J. Cardiol. 102, $383-389$.

Nef, H.M., Mollmann, H., Mollmann, S., Dill, T., Hamm, C.W., Elsasser, A., 2007. Primary stenting of the left main coronary artery with anomalous origin from the right sinus of Valsalva. Int. J. Cardiol. 114, 137-138.

Tejada, J.G., Hernandez, F., Sanchez, I., Martin-Asenjo, R., 2007. Stenting of anomalous left main coronary artery arising from the right sinus of Valsalva: A case report. Int J Cardiol. 119, 266-267.

Yamanaka, O., Hobbs, R.E., 1990. Coronary artery anomalies in 126,595 patients undergoing coronary arteriography. Catheter Cardiovasc Diagn. 21, 28-40. 